\title{
Estudo toxicológico agudo do extrato etanólico de partes aéreas de Jatropha gossypiifolia L. em ratos
}

\author{
Saulo R. Mariz ${ }^{1 *}$, Gilberto S. Cerqueira ${ }^{2}$,Washington C. Araújo ${ }^{3}$, José C. Duarte ${ }^{3}$, Arquimedes \\ F.M. Melo ${ }^{4}$,Hosana B. Santos ${ }^{5}$, Kardilândia Oliveira ${ }^{5}$,Margareth F.F. Melo Diniz, \\ Isac A. Medeiros ${ }^{3}$ \\ 'Departamento de Farmácia, Universidade Federal do Maranhão, Rua 13 de Maio 506, Centro, \\ 65010-600, São Luís, MA, Brasil, \\ ${ }^{2}$ Departamento de Fisiologia e Patologia, Universidade Federal da Paraiba, 58051-970, \\ João Pessoa, PB, Brasil, \\ ${ }^{3}$ Laboratório de Tecnologia Farmacêutica "Prof. Delby Fernandes de Medeiros", Universidade \\ Federal da Paraiba, 58051-970, João Pessoa, PB, Brasil, \\ ${ }^{4}$ Laboratório de Pesquisas Toxicológicas, Departamento de Ciências Farmacêuticas, Universidade Federal de \\ Pernambuco, 50670-901, Recife, PE, Brasil, \\ ${ }^{5}$ Hospital Universitário "Lauro Wanderley", Universidade Federal da Paraíba, 58051-970, \\ João Pessoa, PB, Brasil
}

\begin{abstract}
RESUMO: Este trabalho teve como objetivo a avaliação da toxicidade aguda do extrato etanólico (EE) de partes aéreas de Jatropha gossypiifolia L., espécie vegetal muito usada na medicina popular, apesar de ser catalogada como planta tóxica. Ratos Wistar foram tratados por via oral com doses únicas do extrato $(1,2 \mathrm{~g} / \mathrm{kg} ; 1,8 \mathrm{~g} / \mathrm{kg} ; 2,7 \mathrm{~g} / \mathrm{kg} ; 4,0 \mathrm{~g} / \mathrm{kg}$ e $5,0 \mathrm{~g} / \mathrm{kg})$ e observados por 14 dias. Os principais sinais de toxicidade encontrados, em alguns animais, foram: ptose palpebral, perda de peso e paralisia do trem posterior. Outras alterações significativas ocorreram apenas em machos tratados com a dose de $5 \mathrm{~g} / \mathrm{kg}$ : aumento dos níveis sangüíneos de creatinina, AST, sódio e potássio; diminuição dos níveis de uréia e albumina; leucopenia, além de discretas alterações na coloração e consistência de vísceras. A dose letal mediana $\left(\mathrm{DL}_{50}\right)$ foi superior a $4,0 \mathrm{~g} / \mathrm{kg}$ para machos e maior do que $5,0 \mathrm{~g} / \mathrm{kg}$ para fêmeas. Estes resultados indicam uma toxicidade aguda oral relativamente baixa; contudo, ressaltam a necessidade da realização de estudos toxicológicos de longa duração com o EE de J. gossypiifolia L.
\end{abstract}

Unitermos: Jatropha gossypiifolia, Euphorbiaceae, toxicidade aguda.

\begin{abstract}
Acute toxicological studies of the ethanol extract of the aerial parts of Jatropha gossypiifolia L. in rats". The aim of this work was the assessment of acute oral toxicity of the ethanol extract (EE) of the aerial parts of Jatropha gossypiifolia L. This plant is used in folk medicine despite its classification as a toxic plant. Wistar rats were treated per oralis with single doses of EE $[1.2 \mathrm{~g} / \mathrm{kg} ; 1.8 \mathrm{~g} / \mathrm{kg} ; 2.7 \mathrm{~g} / \mathrm{kg} ; 4.0 \mathrm{~g} / \mathrm{kg}$ and $5.0 \mathrm{~g} / \mathrm{kg}$ (w.b.)] and observed for 14 days. The most important signs of toxicity were: ptosis; reduction of body weight and hindlimb paralysis. Other sgnificant alterations occurred only in males treated with $5.0 \mathrm{~g} / \mathrm{kg}$ (w.b.): increase of creatinine, AST, sodium and potassium seric levels; reduction of urea and albumin; leukopenia and small alterations in color and consistence of viscera. The $\mathrm{LD}_{50}$ value was higher than $4.0 \mathrm{~g} / \mathrm{kg}$ (w.b.) for males and higher than $5.0 \mathrm{~g} / \mathrm{kg}$ (w.b) for females. These results indicate a low oral acute toxicity, in relative terms, however it shows the importance of long-term toxicological studies of the EE from J. gossypiifolia.
\end{abstract}

Keywords: Jatropha gossypiifolia, Euphorbiaceae, acute toxicity.

\section{INTRODUÇÃO}

Nos últimos vinte anos no Brasil, país com a maior diversidade vegetal do mundo, o número de informações sobre plantas medicinais tem crescido apenas $8 \%$ anualmente. Isso mostra que em um país biologicamente tão rico, mas com ecossistemas tão ameaçados, pesquisas com plantas medicinais, e, mesmo tóxicas, deveriam ser mais incentivadas. Afinal, elas poderiam levar à reorganização das estruturas de uso dos recursos naturais (em vista da necessidade de sua extração estar associada aos planos de manejo) e à elevação do PIB, visto que há grande tendência mundial de aumento na utilização de fitoterápicos (Brasil 1998, Falcão et al., 2005; Barbosa-Filho et al., 2005).

A Jatropha gossypiifolia L. é popularmente conhecida no Brasil por vários nomes, tais como, piãoroxo ou pinhão-roxo. Pertence à família Euphorbiaceae; 
gênero Jatropha; subgênero Jatropha; secção Jatropha e subsecção Adenophorae. Apresenta-se como uma árvore de folhas alternas grandes, flores roxas, frutos pequenos e capsulares. Várias partes da planta têm sido usadas na medicina popular para o tratamento de diversas doenças, como: úlceras pépticas, diabetes, neoplasias, diarréias e ainda como cicatrizante e diurético (Pio-Corrêa, 1984; Schvartsman, 1992; Silva, 1998; Mariz et al., 2004).

Alguns usos populares podem ser, em parte, compreendidos pela constituição química, um dos aspectos melhor estudados desta espécie. Substâncias de diversos grupos já foram identificadas como, por exemplo: ácidos orgânicos, alcalóides, diterpenos, esteróides, flavonóides, lignanas e taninos, entre diversos outros constituintes (Subramanian; Nagarajan; Sulochana, 1971; Adesina, 1982; Ogbobe; Akano, 1993; Coe; Anderson, 1996; Das; Anjani, 1999; Mariz et al., 2004).

Tais compostos conferem à $J$. gossypiifolia diversas atividades biológicas úteis na terapêutica, algumas destas já demonstradas experimentalmente: antidiarréico (Silva et al., 2002); antimalárico (Gbeassor et al., 1989); antimicrobiano (Awachie; Ugwu,1997); antiviral (Taylor et al., 1996); moluscicida (Sukumaran; Parashar; Rao, 1995) e sedativo (Adesina, 1982), entre outros (Mariz et al., 2004). Ainda convém destacar que estudos recentes com o extrato etanólico (EE) de partes aéreas de $J$. gossypiifolia indicam que esta espécie, à semelhança de outras do mesmo gênero, possui um significativo potencial hipotensor e vasorelaxante (Abreu et al., 2003).

Entretanto, esta espécie figura entre as principais plantas tóxicas conhecidas em nosso país. Sabe-se que o látex é extremamente cáustico para pele e mucosas. Quando ingerida a planta pode causar distúrbios digestivos e depressores do sistema respiratório e cardiovascular, além de insuficiência renal (Adolf; Opferkuch; Hecker, 1984; Guirola et al., 1992; Schvartsman, 1992; Mengue et al., 2001).

Estudos sobre avaliação de toxicidade de partes de $J$. gossypiifolia usadas na terapêutica têm evidenciado resultados diversos e ainda inconclusivos, tais como: fraca inibição da produção de reticulócitos em coelho (GasperiCampani et al., 1980) e suspeita de correlação positiva com incidência de câncer de esôfago (Hecker, 1984). Por outro lado, tem sido demonstrado que esta planta possui uma $\mathrm{CL}_{50}$ para Artermia salina superior a $1000 \mu \mathrm{g} / \mathrm{mL}$ (Awachie; Ugwu., 1997) e que o tratamento de ratos com o extrato etanólico de partes aéreas de $J$. gossypiifolia por 30 dias, na dose de $500 \mathrm{mg} / \mathrm{kg}$, não produziu alterações bioquímicas e/ou hematológicas nesses animais (Rocha et al., 1995).

Assim, este trabalho se propõe a realizar uma avaliação da toxicidade aguda do EE do pião-roxo com o objetivo de gerar dados que permitam uma análise da relação risco/benefício de um eventual uso terapêutico desta espécie vegetal.

\section{MATERIAL E MÉTODOS}

\section{Coleta do material}

As partes aéreas (folhas e caules) de $J$. gossypiifolia L. foram coletadas no município de Santa Rita (PB) entre os meses de junho e agosto de 2004. O material vegetal foi identificado pelo Herbário "Lauro Pires Xavier" da Universidade Federal da Paraíba (UFPB).

\section{Preparação do extrato etanólico}

As folhas e caules foram desidratados, separadamente, em estufa com ar circulante, a $38^{\circ} \mathrm{C}$ por 72 horas e triturados em moinho tipo Harley. Depois de triturados, os farmacógenos foram misturados e macerados em etanol 95\% à temperatura ambiente, por 72 horas. Em seguida o material foi filtrado e concentrado a vácuo em rotaevaporador, obtendo-se o Extrato Etanólico (EE). A determinação do peso seco desse extrato foi feita pelo método gravimétrico (Farmacopéia Brasileira, 1988). Antes dos experimentos e a partir do peso seco, o EE era diluído com água de modo a se obter soluções em concentrações adequadas para a administração correta das doses. Procedeu-se ainda uma triagem fitoquímica no EE (Matos, 1997).

\section{Animais}

Foram utilizados ratos Wistar albinos (Rattus norvergicus) e adultos. Os machos pesavam entre 250 a $350 \mathrm{~g}$ e as fêmeas eram nulíparas e não grávidas, pesando entre 150 a $250 \mathrm{~g}$. Os animais foram fornecidos pelo Biotério "Prof. Dr. Thomas George" do Laboratório de Tecnologia Farmacêutica (LTF-UFPB). Estes animais foram mantidos em grupos de seis por gaiola sob condições ambientais padronizadas e alimentados com ração e água potável à vontade. A utilização dos animais obedeceu a princípios técnicos e éticos (Andersen et al., 2004; Colégio Brasileiro de Experimentação Animal, 2004).

\section{Ensaios toxicológicos}

Os protocolos experimentais foram orientados pelas normas vigentes em nosso país, por obras de referência e ainda por padronização prévia realizada no Biotério do LTF-UFPB (Larini, 1997; Melo-Diniz, 2000; Barros; Davino, 2003; Brasil, 2004; Jacobson-Kram; Keller, 2001).

Os animais foram tratados por gavagem inicialmente com a dose de $5 \mathrm{~g} / \mathrm{kg}$ do EE de J. gossypiifolia ( $\mathrm{n}=12$ por dose e sexo) e posteriormente com 4 doses inferiores a $5 \mathrm{~g} / \mathrm{kg}$, a saber: $1,2 \mathrm{~g} / \mathrm{kg} ; 1,8 \mathrm{~g} / \mathrm{kg} ; 2,7 \mathrm{~g} / \mathrm{kg}$ e $4,0 \mathrm{~g} / \mathrm{kg}(\mathrm{n}=6$ animais por dose e por sexo).

Durante 14 dias após o tratamento observou- 
se o surgimento de sinais tóxicos gerais usando-se a metodologia proposta por Almeida et al. (1999). Nesse período de observação ainda foram mensurados os seguintes parâmetros: evolução ponderal, consumo de água e alimentos e registro da letalidade. Os consumos de água e ração foram avaliados diariamente e contabilizados por grupo (sexo/dose), registrando-se os valores médios de cada grupo. Os animais sobreviventes foram sacrificados, procedendo-se à coleta de sangue para realização de exames hematológicos e bioquímicos, bem como a ressecção, pesagem e análise macroscópica das vísceras (fígado, rins, coração e pulmões). Também foi feita a análise macroscópica das vísceras dos animais que morreram ao longo do período de observação.

Um grupo controle com 12 animais (6 machos e 6 fêmeas) foi tratado com água, veículo de ressuspensão do extrato.

\section{Análise estatística}

Os resultados numéricos foram expressos em média aritmética ( \pm erro padrão). As diferenças entre os grupos foram determinadas através da Análise de Variância (ANOVA) seguida, quando detectada diferença, pelo teste de múltiplas comparações de Tukey. As análises foram feitas com auxílio do programa GraphPad Prism 3.0. Os resultados foram considerados estatisticamente significativos quando $\mathrm{p}<0,05$ (Gad;Weil, 1989; Graphpad Prism, 1999).

\section{RESULTADOS E DISCUSSÃO}

\section{Obtenção do extrato etanólico (EE)}

O EE obtido apresentou-se como um resíduo escuro, viscoso e higroscópico (rendimento: 7,9\%). A triagem fitoquímica confirmou a presença de taninos, esteróides e flavonóides.

\section{Ensaios toxicológicos}

Entre os animais do grupo controle, tanto não foram observadas alterações orgânicas significativas, quanto seus valores de parâmetros avaliados numericamente encontraram-se de acordo com os valores de referência para os animais do Biotério, obtidos por Melo-Diniz (2000) em padronização prévia.

O surgimento de efeitos tóxicos significativos nos animais tratados com a dose de $5 \mathrm{~g} / \mathrm{kg}$, considerada como dose limite para avaliação toxicológica aguda (Brasil, 2004; Barros; Davino, 2003), nos levou a utilizar outras 4 doses inferiores, na seqüência dos estudos, conforme relatado anteriormente.

Não foram observadas alterações orgânicas dignas de nota, entre os animais que receberam a menor dose $(1,2 \mathrm{~g} / \mathrm{kg})$ do extrato. Essa dose corresponde a 4,8 vezes a maior dose experimental utilizada por Abreu et al. (2003) na demonstração do efeito hipotensor da planta. Este dado informa sobre um limite de segurança do produto em termos de toxicidade aguda, embora de modo não exato.

Em relação aos animais tratados com dose igual ou superior a $1,8 \mathrm{~g} / \mathrm{kg}$, logo nas primeiras 4 horas após o tratamento, foram observados os seguintes sinais gerais de toxicidade: discreta piloereção, prostração, contorções abdominais e aumento da evacuação. A partir do $3^{\circ}$ dia, além da manutenção de piloereção e prostração, surgiram: comportamento estereotipado de autolimpeza ("grooming"); agrupamentos discretos; dispnéia e ptose palpebral. Estes efeitos ocorreram em ambos os sexos. As alterações neurológicas podem ser consideradas como inespecíficas, apesar da predominância de sinais indicativos de ação depressora no Sistema Nervoso Central (SNC), principalmente: prostração e ptose palpebral. Os distúrbios gastrintestinais são coerentes com a clínica de intoxicação já relatada para outra espécie do mesmo gênero, a Jatropha curcas (Schvarstman, 1992).

Entre os animais que morreram, a perda gradativa de peso e a paralisia do trem posterior (figura 1) foram sinais anteriores ao óbito. Esta informação pode ser importante em estudos de causa mortis ou de mecanismos de toxicidade. Parece que a contração da musculatura lisa abdominal, observada em altas doses, provocaria não somente cólicas e diarréias, o que prejudica a ingesta alimentar (figura 2), como também resultaria em danos neuro-musculares graves causando paralisia em toda a região posterior do animal (figura 1).

Apenas entre machos sobreviventes à maior dose, o extrato administrado reduziu o consumo de alimentos de modo significativo (figura 2). Esta dose de $5 \mathrm{~g} / \mathrm{kg}$ ainda reduziu a evolução ponderal de fêmeas, do $6^{\circ}$ ao $11^{\circ}$ dia após o tratamento (figura 3 ), sem alterar a ingestão de alimentos destes animais (figura 2), o que poderia sugerir uma linha de investigação interessante se não fosse a alta dose empregada ( $5 \mathrm{~g} / \mathrm{kg})$.

Alterações bioquímicas e hematológicas estatisticamente significativas foram observadas apenas entre os machos tratados com a maior dose $(5 \mathrm{~g} / \mathrm{kg})$, conforme agrupado na Tabela I, o que retrata a maior sensibilidade dos machos ao extrato.

Inicialmente tem-se uma hipoalbuminemia, constatada tanto pela diferença estatisticamente significativa entre tratados e controles quanto pela significância clínica dessa alteração considerando-se que o valor de albumina dos animais tratados foi $12,9 \%$ menor que o limite inferior de referência desse parâmetro $(3,1$ $\mathrm{g} / \mathrm{dL})$. Esse quadro pode ser compreendido pela redução na ingestão de alimentos (figura 2). Todavia, tal condição clínica também pode decorrer de dano hepático grave, considerando-se que a síntese dessa proteína ocorre exclusivamente nas células hepáticas. Essa hipótese de agravo hepático é reforçada pelo aumento da aspartato 


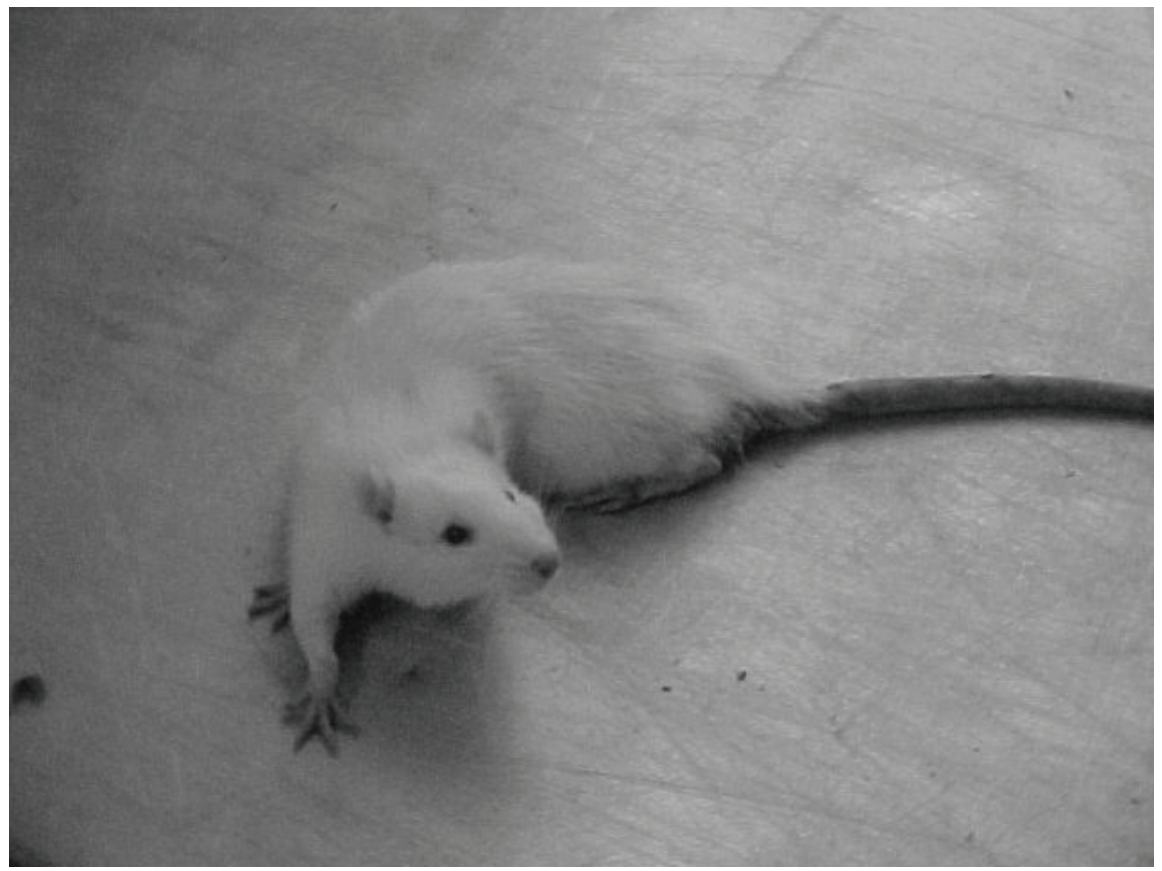

Figura 1. Imagem de arraste do trem posterior em rato Wistar tratado com EE de $J$. gossypiifolia (dose $\geq 1,8 \mathrm{~g} / \mathrm{kg}$ ).

aminotransferase (AST) em 21,6\% do valor máximo de referência ( $89 \mathrm{U} / \mathrm{L})$, fato que também pode significar distrofia muscular (inclusive no músculo cardíaco) e embolia pulmonar, além de outras condições clínicas (Kaneko, 1996; Ravel, 1997; Melo-Diniz, 2000).

A hipouremia observada, valores $35,3 \%$ menores que a referência mínima $(51 \mathrm{mg} / \mathrm{dL})$, também corrobora o indício de dano hepático grave; entretanto, esse parâmetro é mais usado em associação com a dosagem de creatinina, como prova de função renal. Nos animais tratados com o extrato $(5 \mathrm{~g} / \mathrm{kg})$, ocorreu elevação da creatinina em $10 \%$ da referência máxima $(0,6 \mathrm{mg} / \mathrm{dL})$, o que pode significar dano renal grave considerando-se a maior velocidade de depuração da creatinina em relação à uréia (Kaneko, 1996; Ravel, 1997; Melo-Diniz, 2000).

$\mathrm{O}$ aumento do sódio $\left(\mathrm{Na}^{+}\right)$, apesar de estatisticamente significativo, foi clinicamente discreto, pois ultrapassou apenas em $1,6 \%$ o valor de referência limítrofe $(140 \mathrm{mEq} / \mathrm{L})$ para os animais do biotério. Discretas elevações de $\mathrm{Na}^{+}$podem refletir desequilíbrio hidro-eletrolítico, como o gerado por diarréias. No entanto, para o potássio $\left(\mathrm{K}^{+}\right)$a elevação em relação ao limite superior dos valores de referência $(5,4 \mathrm{mEq} / \mathrm{L})$ foi de $31,4 \%$. Essa hiperpotassemia pode significar, além de desequilíbrio hidro-eletrolítico, também alto grau de dano neuro-muscular (Kaneko, 1996; Ravel, 1997; MeloDiniz, 2000).

A única alteração hematológica observada foi uma forte redução na contagem global de leucócitos (49,1\% em relação à referência mínima que é de 6,8 $10^{3} / \mathrm{mm}^{3}$ ) sem modificações na contagem diferencial, o que caracteriza uma leucopenia inespecífica (Jain, 1993; Melo-Diniz, 2000).

O exame macroscópico das vísceras (coração, pulmão, fígado e rins) dos animais sobreviventes não detectou alterações dignas de nota. Apenas em alguns dos animais tratados com a dose de $5 \mathrm{~g} / \mathrm{kg}$ e que morreram ao longo do experimento (machos), foram encontradas alterações de coloração e consistência em coração, pulmão, fígado e intestino, o que ressalta a importância da realização de exames histopatológicos nessas vísceras para uma melhor avaliação de possíveis efeitos inflamatórios e necrosantes.

Observou-se que o extrato apenas produziu mortes em machos e nas duas maiores doses empregadas: $4,0 \mathrm{~g} / \mathrm{kg}(16,6 \%)$ e $5 \mathrm{~g} / \mathrm{kg}(66,6 \%)$. As mortes ocorreram do terceiro ao décimo dia após o tratamento o que aponta para uma absorção bastante lenta do extrato no trato gastrintestinal, fato compreensível pela ocorrência de intensas diarréias, principalmente nas doses mais altas, o que, por certo, funcionou como um mecanismo de defesa do organismo, aumentando a excreção do produto e retardando o efeito letal do extrato.

A análise da letalidade em cada grupo experimental indica que a dose letal mediana $\left(\mathrm{DL}_{50}\right)$ está entre 4,0 e $5 \mathrm{~g} / \mathrm{kg}$ para machos e é superior a $5 \mathrm{~g} / \mathrm{kg}$ em fêmeas, valores bem acima da dose limite para avaliação desse parâmetro que é de $2 \mathrm{~g} / \mathrm{kg}$, segundo alguns autores (Larini, 1997; Barros; Davino, 2003). Sendo assim, a determinação de um valor de $\mathrm{DL}_{50}$ mais exato, através de novos experimentos, não seria mero preciosismo, mas sim, um erro em termos de bioética animal (Andersen et 


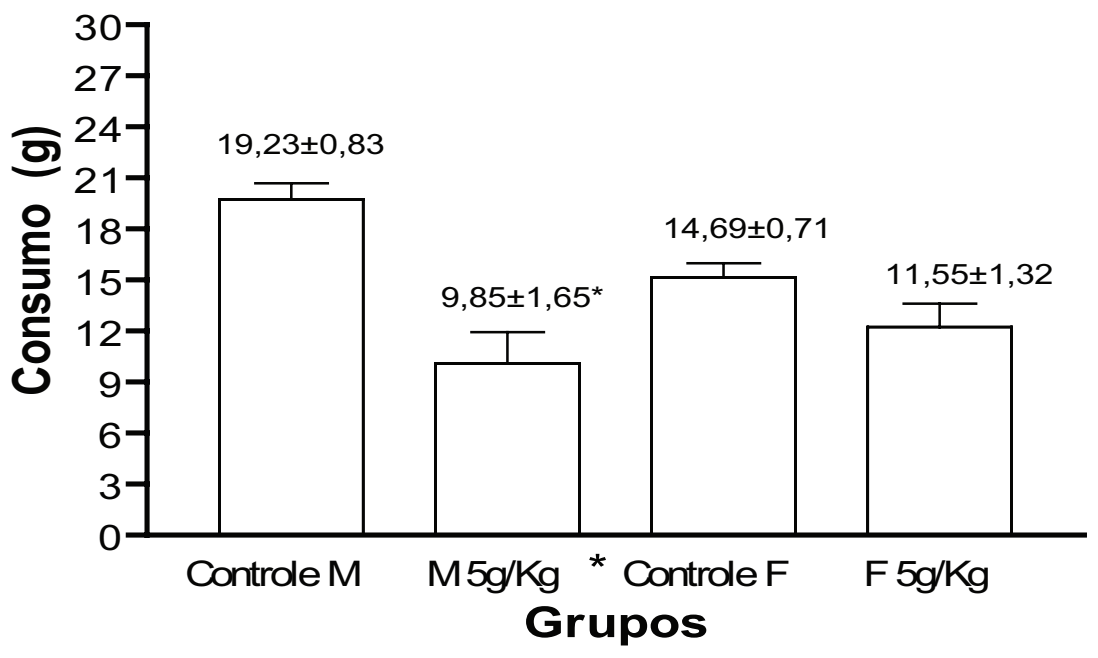

Figura 2. Consumo de alimentos (média \pm e.p.m.) entre ratos Wistar após tratamento com EE de $J$. gossypiifolia $(5 \mathrm{~g} / \mathrm{kg}$ ). Legenda: M (machos); F (fêmeas). *Valor estatisticamente diferente do grupo controle (ANOVA seguido de Tukey, $\mathrm{p}<0,05$ ).

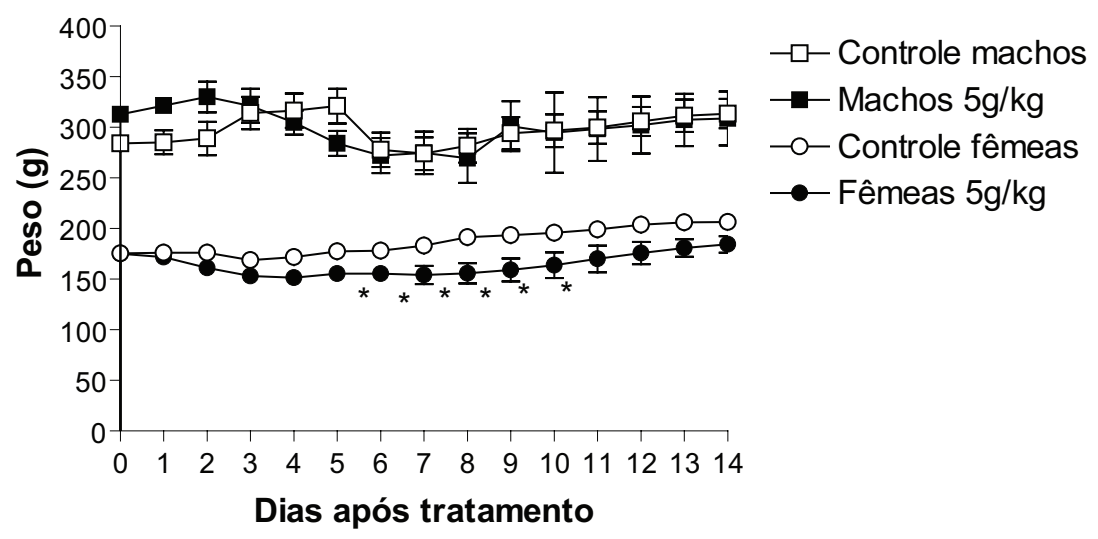

Figura 3. Evolução ponderal (média \pm e.p.m.) de ratos Wistar tratados com EE de $J$. gossypiifolia $(5 \mathrm{~g} / \mathrm{kg})$. *Valores estatisticamente diferentes do grupo controle (ANOVA seguido de Tukey, $\mathrm{p}<0,05)$.

Tabela 1. Parâmetros sangüíneos de ratos Wistar machos, alterados significativamente (ANOVA seguido de Tukey, p $<0,05$ ) pelo tratamento com dose única (v.o.) do EE de J. gossypiifolia L (5 g/kg).

\begin{tabular}{c|c|c|c}
\hline Parâmetros & \multicolumn{3}{|c}{ Grupo } \\
\hline & Controle & Tratado & Referência* \\
Albumina $(\mathrm{g} / \mathrm{dL})$ & $3,3 \pm 0,09$ & $2,7 \pm 0,02$ & 3,1 a 3,5 \\
Uréia $(\mathrm{mg} / \mathrm{dL})$ & $56,0 \pm 2,3$ & $33,0 \pm 0,8$ & 51,0 a 61,0 \\
Creatinina $(\mathrm{mg} / \mathrm{dL})$ & $0,5 \pm 0,04$ & $0,66 \pm 0,02$ & 0,4 a 0,6 \\
$\mathrm{Na}^{+}(\mathrm{mEq} / \mathrm{L})$ & $137,0 \pm 1,41$ & $142,33 \pm 0,27$ & 134,0 a 140,0 \\
$\mathrm{~K}^{+}(\mathrm{mEq} / \mathrm{L})$ & $5,1 \pm 0,13$ & $7,1 \pm 0,16$ & 4,8 a 5,4 \\
$\mathrm{AST}(\mathrm{U} / \mathrm{L})$ & $78,0 \pm 5,4$ & $108,3 \pm 6,2$ & 67,0 a 89,0 \\
Leucócitos $\left(10^{3} / \mathrm{mm}^{3}\right)$ & $8,4 \pm 0,75$ & $3,4 \pm 0,47$ & 6,8 a 10 \\
\hline
\end{tabular}

*Valores preconizados por Melo-Diniz (2000). Os valores dos grupos (tratado e controle) representam a média \pm e.p.m. $\mathrm{Na}^{+}$(sódio); $\mathrm{K}^{+}$(potássio); AST (aspartato aminotransferase). 
al. 2004).

A indicação de relativa segurança de exposição, aguda e em dose única, ao produto estudado é reforçada pelo alto valor da dose máxima não letal (DMNL) estimado nesse estudo (machos $=2,8 \mathrm{~g} / \mathrm{kg}$ e fêmeas $=5 \mathrm{~g} / \mathrm{kg}$ ). Esse indicador tem sido proposto como sendo mais útil que a $\mathrm{DL}_{50}$ para a avaliação da relação risco/segurança de um produto pelo fato de usar a não ocorrência de mortes (mais grave dos efeitos tóxicos) como critério analítico (Larini, 1997).

Os resultados indicam uma toxicidade aguda oral relativamente baixa para o EE de J. gossypiifolia, considerando-se que: uma dose relativamente alta $(1,2$ $\mathrm{g} / \mathrm{kg}$ ) não causou alterações orgânicas significativas; os efeitos tóxicos mais graves só foram observados em machos tratados com a maior dose experimental $(5 \mathrm{~g} / \mathrm{kg})$, dose essa 20 vezes maior que a provável dose terapêutica do produto e ainda que indicadores clássicos de avaliação de toxicidade, como $\mathrm{DL}_{50}$ e DMNL, foram estimados em valores elevados.

Apesar disso, não devemos ignorar que os indícios de toxicidade neurológica, hepática e renal, foram obtidos com doses que, apesar de elevadas, eram únicas, como convinha aos objetivos deste estudo. Isso suscita o questionamento sobre uma eventual permanência desses efeitos e/ou o surgimento de outros quando de um tratamento prolongado com o produto em questão.

\section{CONCLUSÕES}

O extrato etanólico de J. gossypiifolia L. apresentou toxicidade aguda oral relativamente baixa em ratos Wistar. Apesar disso, em função das alterações orgânicas observadas, sugere-se a continuidade dos estudos mediante a avaliação da toxicidade crônica do produto.

\section{AGRADECIMENTOS}

Os autores agradecem à Comissão de Aperfeiçoamento de Pessoal de Ensino Superior (CAPES) que, através do Programa de Qualificação Interinstitucional (PQI), tem financiado esta pesquisa. Ainda agradecem à $\operatorname{Prof}^{\mathrm{a}} \operatorname{Dr}^{\mathrm{a}}$ Maria de Fátima Agra pela identificação da espécie e à Prof ${ }^{a} \operatorname{Dr}^{a}$ Temilce Simões de Assis pela revisão do abstract.

\section{REFERÊNCIAS}

Abreu IC, Marinho ASS, Paes AMA, Freire SMF, Olea RSG, Borges MOR, Borges ACR 2003. Hypotensive and vasorelaxant effects of the ethanolic extract from Jatropha gossypiifolia L. in rats. Fitoterapia 74: 651-657

Adesina SK 1982. Studies on some plants used as anticonvulsants in Amerindian and African tradicional medicine. Fitoterapia 53:147-162.

Adolf W, Opferkuch HJ, Hecker E 1984. Irritant phorbol derivatives from four Jatropha species. Phytochemistry 23: 129-132.

Almeida RN, Falcão ACGM, Diniz RST, Quintans-Júnior LJ, Polari RN, Barbosa-Filho JM, Agra MF, Duarte JC, Ferreira CD, Antoniolli AR, Araújo CC 1999. Metodologia para avaliação de plantas com atividade no sistema nervoso central e alguns dados experimentais. Rev Bras Farm 80: 72-76.

Andersen ML, D'Almeida V, Ko GM, Kawakami R, Martins PJF, Magalhães LE, Tufik S 2004. Principios Éticos e Práticos do Uso de Animais de Experimentação. São Paulo: UNIFESP.

Awachie PIA, Ugwu FO 1997. Preliminary investigation of the antimicrobial and brine shrimp lethality properties of some Nigerian medicinal plants. Int J Pharmacogn 35: 338-343.

Barbosa-Filho JM, Vasconcelos THC, Alencar AA, Batista LM, Oliveira RAG, Guedes DN, Falcão HS, Moura MD, Diniz MFFM, Modesto-Filho J. 2005. Plants and their active constituents from South, Central, and North America with hypoglycemic activity. Rev Bras Farmacogn 15: 392-413.

Barros SBM, Davino SC. 2003. Avaliação da Toxicidade. In: S. Oga . Fundamentos de Toxicologia. 2.ed. São Paulo: Atheneu. p.57-67.

Brasil 1998. Primeiro relatório nacional para a conservação sobre diversidade biológica: Brasil. Ministério do Meio Ambiente, dos Recursos Hídricos e da Amazônia Legal, Brasília.

Brasil 2004. Ministério da Saúde. Agência Nacional de Vigilância Sanitária (ANVISA). Resolução - RE $n^{\circ}$ 90/2004. Normas para estudos toxicológicos de produtos fitoterápicos. Diário Oficial [da] República Federativa do Brasil, Poder Executivo, Brasilía, DF, 12 de março de 2004.

Coe FG, Anderson GJ 1996. Screening of medicinal plants used by Garífuna of Eastern Nicaragua for bioactive compounds. J. Ethnopharmacol 53: 29-50.

Colégio Brasileiro de Experimentação Animal (COBEA). Legislação e Ética. Resolução 592. Disponível em: $<$ http://www.meusite.com.br/COBEA/>. Acesso em 01 Ago. 2004.

Das B, Anjani G 1999. Gossypidien, a lignan from stems of Jatropha gossypifollia. Phytochemistry 51: 115117.

Falcão HS, Lima IO, Santos VL, Dantas HF, Diniz MFFM, Barbosa-Filho JM, Batista LM 2005. Review of the plants with anti-infl ammatory activity studied in Brazil. Rev Bras Farmacogn 15: 381-391.

Farmacopéia Brasileira 1988. $4^{\mathrm{a}}$ ed. São Paulo: Atheneu Editora. v.2.9.

Gad SC, Weil CS 1989. Statistics for Toxicologists. In: Hayes AW (Ed.). Principles and Methods of Toxicology. New York: Raven Press, p.435-483.

Gasperi-Campani A, Barbieri L, Morelli P, Stirpe F 1980. Seed extracts inhibiting protein synthesis in vitro. Biochem J 186: 439-441.

Gbeassor M, Kossou Y, Amegbo K, De Souza C, Koumaglo K 1989. Antimalarial effects of eight african medicinal plants. J. Ethnopharmacol 25: 115-118.

Graphpad Prism 1999. User's Guide Version 3.0: the fast, organized way to analyze and graph scientific data. San Diego. Disponível em: <http:www.graphpad. 
com>. Acesso em 23 mai. 2004.

Guirola L, Garcia G, Torrealba A, Espinoza OB, Irastorza IM, Ramirez MS 1992. Acute renal failure from the ingestion of toxic plants. Vet Hum Toxicol 34: 548.

Hecker E 1984. Co-carcinogenic diterpene esters as principal risk factors in local life style esophageal cancer in Curaçao. Acta Pharmacol Toxicol 55: 148-153.

Jacobson-Kram D, Keller KA 2001. Toxicology Testing Handbook. New York: Marcel Dekker.

Jain CN 1993. Essentials of Veterinary Hematology. Philadelphia: Lea \& Febiger.

Kaneko JJ 1996. Clinical Biochemistry of Domestic Animals. San Diego: Academic Press.

Larini L 1997. Avaliação Toxicológica. In: Larini L. Toxicologia. 3ed. São Paulo: Manole. p.43-58.

Mariz SR, Medeiros IA, Melo-Diniz MFF, Borges ACR, Borges MOR, Cerqueira GS, Araújo WC 2004. Potencial terapêutico e risco toxicológico de Jatropha gossypiifolia L.: uma revisão. XVIII Simpósio de Plantas Medicinais do Brasil. Manaus, Brasil.

Matos FJA 1997. Introdução a Fitoquímica Experimental, $2^{a}$ ed. Fortaleza: Edições UFC.

Melo-Diniz MFF 2000. Estudos de toxicidade pré-clínica de extratos de folhas de Cissampelos sympodialis Eich. 147 p. Tese de Doutorado - Programa de PósGraduação em Produtos Naturais, Universidade Federal da Paraíba.

Mengue SS, Mentz LA, Schenzel EP 2001. Uso de plantas medicinais na gravidez. Rev Bras Farmacogn 11: 21-35.

Ogbobe O, Akano V 1993. The physico-chemical properties of the seed and seed oil of Jatropha gossipifolia. Plant Food Hum Nutr 43: 197-200.

Pio-Corrêa M 1984. Dicionário de Plantas Úteis do Brasil $e$ das Exóticas Cultivadas. Rio de Janeiro: Ministério da Agricultura - Instituto Brasileiro de Desenvolvimento Florestal v.5, p.485.

Ravel R 1997. Laboratório Clínico: aplicações clínicas dos achados laboratoriais. Rio de Janeiro: Guanabara Koogan.

Rocha MO, Borges ACR, Azevedo ACF, Castro SFL 1995. Avaliação toxicológica do tratamento sub-crônico com extrato da Cecropia adenopus Mart., Sechium edule e Jatropha gossypiifolia L. nos compostos bioquímicos do sangue. $47^{a}$ Reunião Anual da $S B P C$, São Luís. Brasil.

Schvartsman S 1992. Plantas Venenosas e Animais Peçonhentos. São Paulo: Sarvier.

Silva SN, Ribeiro RM, Abreu IC, Castro AS, Borges MOR, Borges ACR, Freire SMF 2002. Antagonismo não-competitivo de frações obtidas de Jatropha gossypiifolia L. (Pião-Roxo) no músculo liso intestinal. XIV Seminário de Iniciação Científica da Universidade Federal do Maranhão (SEMICUFMA). São Luis. Brasil.

Silva SI 1998. Euphorbiaceae da Caatinga: distribuição de espécies e potencial oleaginoso. 132p. Tese de Doutorado - Instituto de Biociências, Universidade de São Paulo.

Subramanian SS, Nagarajan S, Sulochana N 1971. Flavonoids of the leaves of Jatropha gossypifolia. Phytochemistry 10: 1690

Sukumaran D, Parashar BD, Rao KM 1995. Toxicity of Jatropha gossypiifolia and Vaccaria pyramidata against freshwater snails vectors of animal schistosomiasis. Fitoterapia 66: 393-398.

Taylor RSL, Hudson JB, Manandhar NP, Towers GHN 1996. Antiviral activities of medicinal plants of southern Nepal. J Ethnopharmacol 53: 97-104.

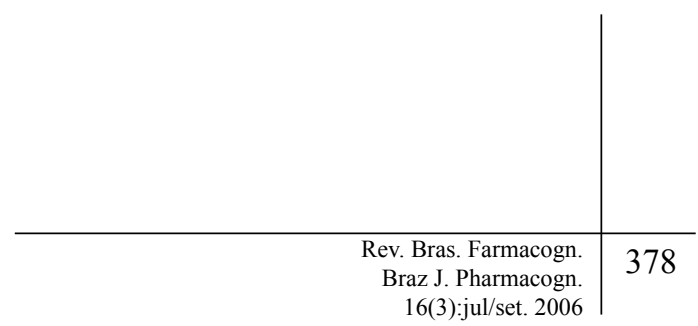

